It may therefore be concluded that cysteine causes apparent inhibition of ChA by consumption of the acetylCoA substrate by non-enzyme transacetylation. The rate of $S$-acetylcysteine formation, both in the presence of enzyme and the complete incubation mixture, and in a mixture of acetyl-CoA and cysteine (Fig. 1), paralleled the reduction in the rate of $\mathrm{ACh}$ formation.

J. Crispin Smith

RichaRd B. WeISKOPF

Anesthesiology Research Laboratory,

Montefiore Hospital and Medical Center,

New York.

Received June 2; revised August 7, 1967.

1 Morris, D., Hebb, C., and Bull, G., Nature, 209, 914 (1966).

${ }^{2}$ Morris, D., J. Neurochem., 14, 19 (1967).

${ }^{3}$ McCaman, R. E., and Hunt, J. M., J. Neurochem., 12, 253 (1965).

4 Potter, L. T., and Murphy, W., Biochem. Pharmacol., 16, 1386 (1967).

\section{Use of Urine to Serum Fluoride Concentration Ratios to confirm Serum Fluoride Analyses}

IN 1966 I published ${ }^{1}$ results showing that the normal concentration of fluoride in human serum is about onetenth the generally accepted value-that of Singer and Armstrong. Part of the evidence supporting my results was the "at least five-fold discrepancy" that could be shown in their data ${ }^{2}$ which is most easily explained by an error in the measurement of the serum fluoride. The discrepancy involves the ratio of urine to plasma fluoride concentrations when determined by fluorine-18 as compared with stable fluoride analyses. Because the concentration of fluoride in urine is well established in contrast to that in serum where analysis is much more difficult, it is reasonable to question the latter when there is a discrepancy. Singer and Armstrong ${ }^{3}$, however, in their recent rebuttal, think that $I$ came to erroneous conclusions because I directed attention to only two of their analyses, which they now consider aberrant.

In summarizing the data as a whole they calculate that the average concentration of fluoride in the urine from one subject (C.H.C.) was 90 umolar (1.7 p.p.m.) and from the other (W.D.A.), $179 \mu$ molar (3.4 p.p.m.). They state that these concentrations are reasonable because $I$ indicated that up to $150 \mu$ molar (3 p.p.m.) ${ }^{1}$ might be expected in the two samples where there was a very concentrated urine. Such a comparison completely overlooks the effect of the flow rate of urine on the expected concentration of fluoride. In the case of subject C.H.C., $1,888 \mathrm{ml}$. of urine was collected in $321 \mathrm{~min}$, or $5.9 \mathrm{ml} . / \mathrm{min}$, in contrast to the 0.42 and $0.57 \mathrm{ml}$./min of the two "aberrant" samples. If the rate of flow of urine had no effect on clearance of fluoride from the blood then there would be a direct inverse relationship between concentration and rate of flow. Because the average rate of flow is increased ten-fold over the "aberrant" samples, the expected concentration in the urine for the samples as a whole would be one-tenth as much, or up to $15 \mu$ molar $(0 \cdot 3$ p.p.m.) rather than $150 \mu$ molar (3.0 p.p.m.). In subject C.H.C., increasing the rate of flow twenty-fold doubled the clearance of fluorine-18, so there would be some increase in the expected concentration, but not to more than 23 umolar $(0.43$ p.p.m.)

My original estimate of up to $150 \mu$ molar (3.0 p.p.m.) fluoride for the two "aberrant" samples was based on observations" of persons drinking slightly different amounts of water than those used by Carlson et al. ${ }^{2}$. In order to substantiate better the expected urine fluoride concentration in the conditions used by Carlson, Armstrong and Singer, I ingested fluoride and water in the same pattern and collected $1,025 \mathrm{ml}$. of urine in 255 min or $4.0 \mathrm{ml} . / \mathrm{min}$. After thoroughly mixing the urine, five samples were analysed by Singer and Armstrong's $\operatorname{method}^{5}$ and $21 \pm 1.5 \mu$ molar $(0.40$ p.p.m.) fluoride were found. The urine collected before any water was ingested showed $78+8 \mu$ molar (1.47 p.p.m.) fluoride by the same analysis. Analysis of the same samples by my method gave mean values within 3 per cent of those analysed by Singer and Armstrong's method.

If my flow rate had been the same as that of subject C.H.C. then the urine fluoride concentration would have been lower, possibly as low as $14 \mu$ molar $(0.27$ p.p.m.). Thus a four- to six-fold discrepancy exists between their calculated concentration ( $90 \mu$ molar) and what I measured. This does not differ much from the "at least five-fold discrepancy" that I originally pointed out using just the two "aberrant" samples. No data on the volume of urine are given for the other subject (W.D.A.) so a comparable analysis cannot be made, but there would appear to be at least as great a discrepancy, for the concentration as calculated by Singer and Armstrong is twice as great as that for C.H.C. with the same range of urine flow rates. As indicated, the most logical explanation of this discrepancy is to assume that their serum fluoride values are in error. Certainly, they are not measuring what the kidney sees as fluoride.

There is a simple way to show what the normal serum fluoride should be in humans drinking fluoridated water. The average data for clearance of fluorine-18 for all Singer and Armstrong's samples with a flow rate of less than $2 \mathrm{ml} . / \mathrm{min}$, which involves four samples for each subject, are 56 and $49 \mathrm{ml}$. plasma cleared $/ \mathrm{min}$ for the two subjects. If a person is exereting $50 \mu$ molar (1 p.p.m.) fluoride in his urine at a flow rate of $1 \mathrm{ml} . / \mathrm{min}$ then the plasma fluoride concentration must be $0 \cdot 9-1 \cdot 0$ mmolar $(0.018$ to 0.02 p.p.m.), which is very close to the 0.7 umolar I reported.

The inability of Singer and Armstrong ${ }^{5}$ to reproduce my fusion data certainly cannot be taken as a refutation of my findings ${ }^{1}$. First, they fused sodium hydroxide rather than converting it to the carbonate-chloride salt. This makes a difference in the fusion temperature because pure sodium hydroxide fuses at $318^{\circ} \mathrm{C}$ while the carbonate fuses at $851^{\circ} \mathrm{C}$. Second, they only examined serum from one individual and a single individual cannot be assumed to be representative of normal.

The accurate determination of the ratio of fluoride in the urine to serum by means of fluorine-18 has made it possible to confirm measurements of serum fluoride concentration of only 0.1 umolar $(0.002$ p.p.m.) in pups that have been raised on a milk diet. Extension of this principle makes it possible to estimate serum fluoride concentrations as low as $0.01 \mu$ molar, if the need should arise.

Donald R. TAves

Department of Radiation Biology and Biophysics,

University of Rochester,

New York.

Received June 12, 1967.

1 Taves, D. R., Nature,211, 192 (1966).

Carlson, C. H., Armstrong, W. D., and Singer, L., Proc. Soc. Exp. Biol. and Med., 104, 235 (1960).

${ }^{3}$ Singer, L., and Armstrong, W. D., Nature, 214, 1161 (1967).

${ }^{4}$ Zipkin, I., and Leone, N. C., Amer. J. Pub. Health, 47, 848 (1959).

${ }^{3}$ Singer, L., and Armstrong, W. D., Anal. Biochem., 10, 495 (1965).

\section{Traumatic Acid : an Accelerator of Abscission in Cotton Explants}

WE have been investigating the physiology of bud abscission induced by the feeding action of lygus bugs, Lygus hesperus Knight, because explants are used for biological assays ${ }^{1}$. In this bioassay, various extracts are applied in agar to the stumps of the cotyledonary petioles and the time for 50 per cent of the petioles to abscise is used for comparative purposes. In the course of these experi- 